\title{
LA CRIMINALIZACIÓN DE SENEGALESES EN LA CIUDAD DE BUENOS AIRES, EN EL PERÍODO 2015-2019. UN ACERCAMIENTO DESDE LA INTERSECCIONALIDAD
}

Jeremías Pérez RABASA ${ }^{1}$

RESUMEN: El presente texto es un análisis de la migración senegalesa en la Ciudad Autónoma de Buenos Aires. El interés de este estudio es la criminalización a la que fueron sometidos con la asunción del gobierno liberal-conservador de la Alianza Cambiemos. Se señala que la criminalización de este colectivo es la intersección de diferentes discriminaciones a la que son sometidos por el Estado, siendo, los factores más importantes, los cambios normativos en materia migratoria, la persecución callejera por parte de las fuerzas de seguridad, la violencia de estos en el abordaje y la normativa judicial aplicada, que promueven las multinacionales. Las reflexiones que se presentan son el resultado parcial de una investigación que realiza este autor en el marco de una beca doctoral.

PALABRAS CLAVE: senegalese; Ciudad de Buenos Aires; criminalización; interseccionalidad.

THE CRIMINALIZATION OF SENEGALESE IN THE CITY OF BUENOS AIRES, IN THE PERIOD 2015-2019. AN APPROACH FROM THE INTERSECTIONALITY

ABSTRACT: This paper is an analysis of Senegalese migration in the Autonomous City of Buenos Aires. The particular interest of this study is the criminalization to which they were subjected with the inauguration of the liberal-conservative government of Alianza Cambiemos. It is pointed out that the criminalization of this group is the intersection of

1 Magister(CONICET-UNLa). ORCID: https://orcid.org/0000-0003-1304-6887. jperezrabasa@gmail.com. 
different discriminations to which they are subjected by the State, the most important factors being regulatory changes in immigration matters, street persecution by the security forces, violence against them in the approach and the applied judicial regulations, promoted by multinationals. These presented reflections are a partial result of an investigation carried out by this author within the framework of a PhD scholarship.

KEYWORDS: senegalese; City of Buenos Aires; criminalization; intersectionality.

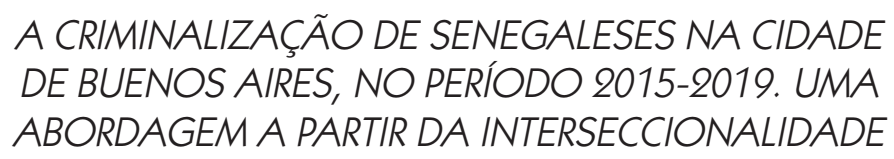

RESUMO: Este texto é uma análise da migração senegalesa na Cidade Autônoma de Buenos Aires. $O$ interesse particular deste estudo é a criminalização a que eles foram submetidos com a posse do governo liberal-conservador Alianza Cambiemos. Salienta-se que a criminalização desse grupo é o cruzamento de diferentes discriminaçôes a que estão sujeitos pelo Estado, sendo os fatores mais importantes as mudanças regulatórias em matéria de imigração, perseguição nas ruas pelas forças de segurança, violência contra estes na abordagem e nas regulamentaçôes judiciais aplicadas, promovidas pelas multinacionais. As reflexōes que se apresentam são o resultado parcial de uma investigaçẫo realizada por este autor no âmbito de uma bolsa de doutoramento.

PALAVRAS-CHAVE: senegaleses; Cidade de Buenos Aires; criminalização; interseccionalidade.

\section{Introducción}

El presente texto es el resultado parcial de una investigación enmarcada en un proyecto doctoral que realiza este autor como becario del CONICET ${ }^{2}$. Los análisis que se exponen son producto de un trabajo que lleva 3 años en ejecución. El interés de este estudio es particularmente el proceso de criminalización de los senegaleses en la Ciudad Autónoma de Buenos Aires, en el período 2015-2019,

\footnotetext{
2 El Consejo Nacional de Investigaciones Científicas y Técnicas (CONICET), es el principal organismo dedicado a la promoción de la ciencia y la tecnología en la Argentina, dependiente del Ministerio de Ciencia, Tecnología e Innovación de la Nación.
} 
a partir de los diferentes cambios en la política migratoria con la asunción de un gobierno liberal-conservador. En este texto se presenta la intersección de diferentes discriminaciones hacia el colectivo senegalés y como estas operan en la construcción de un "ellos" criminal que funciona como justificativo de la violencia estatal. Para abordar esta cuestión, además de un exhaustivo repaso por los trabajos académicos sobre la particularidad de la migración africana en Argentina, se analizaron los informes producidos por el Sistema Internacional de Derechos Humanos en este período para el país, la ley No 25.871 (ARGENTINA, 2003), la Disposición $N^{\circ} 2$ de la Dirección Nacional de Migraciones (ARGENTINA, 2013) sobre regularización de migrantes senegaleses, el Decreto de Necesidad y Urgencia No 70 del año 2017 (ARGENTINA, 2017), los informes del Ministerio Público Fiscal de la Ciudad Autónoma de Buenos Aires en este período (BUENOS AIRES, 2018c), el informe del año 2017 sobre senegaleses del Ministerio Público de la Defensa de la Ciudad Autónoma de Buenos Aires (BUENOS AIRES, 2017a), el informe del 2018 sobre violencia contra senegaleses de la Defensoría del Pueblo de la Ciudad Autónoma de Buenos Aires (BUENOS AIRES, 2018a), las denuncias públicas realizadas por el Centro de Estudios Legales y Sociales (CELS, 2018) y Amnistía Internacional (AMNISTIA INTERNACIONAL, 2017), el informe del año 2019 sobre venta callejera de la Cámara Argentina de Comercio y Servicios (CAC, 2019), y diez notas periodísticas de diarios nacionales que dan cuenta de la situación. Se establecen, en primer lugar, a modo de orden del texto algunos conceptos teóricos desde los cuales se analiza el fenómeno en el apartado denominado "herramientas conceptuales”. Allí se desarrolla el concepto de interseccionalidad y la importancia de aplicación en este caso concreto. En el segundo apartado se explican brevemente algunas características de la comunidad senegalesa migrante en Argentina. En el tercer apartado denominado "Criminalización interseccional" se desarrolla el caso particular desde cuatro aristas distintas, pero interconectadas, la normativa migratoria, la persecución por perfil racial, la violencia en la intervención de las fuerzas de seguridad y algunos elementos relativos al trabajo que desarrolla la mayoría de la comunidad senegalesa en el país. Finalmente, en el último apartado se exponen las palabras finales.

\section{Herramientas conceptuales}

La criminalización de los senegaleses en la Ciudad Autónoma de Buenos Aires es un proceso donde intersectan diferentes formas de discriminación. Se fundamentará a lo largo del trabajo que en este caso opera la xenofobia, el racis- 
mo, la discriminación de clase y la violencia contra los hombres subalternos (MENJIVAR OCHOA, 2017), pero no como una sumatoria de discriminaciones, sino como una intersección de estas. La concepción de las migraciones como problema de seguridad se presenta como tensión desde la propia concepción de los Estados nación en la búsqueda de afirmación de la soberanía (ŽIŽEK, 2016). No obstante, en el contexto actual, estos procesos se han transformado notablemente (BHABHA, 2013). En este sentido, la criminalización del migrante es el proceso de construcción de estos como una amenaza de la cual hay que defenderse, sea con legislación migratoria dura, detenciones o deportaciones o con otras prácticas que pueden ser incluso administrativas que clasifican migrantes y lo relacionan con el delito (BIGO, 1998). Es decir, según de donde venga será más o menos delincuente; de lo que nunca se podrá desligar el migrante es de la sospecha. Pero, además, el arbitrario tratamiento del Estado a ciertos migrantes como si fuesen criminales no está sujeto a una acción determinada del mismo, sino que la definición de a quién el Estado "acepta” y a quién "rechaza" está tomada previamente, antes de la llegada del sujeto migrante en sí. Sobre las clasificaciones y jerarquías en el proceso de criminalización, la criminología crítica hace foco en las relaciones de poder que estructuran las relaciones sociales (BARATTA, 1979), incluso algunos autores señalan directamente a la raza y la clase "(...) como ideologías justificadoras de las relaciones de poder actuales, que además se retroalimentan y refuerzan mutuamente" (MONCLÚS MASO, 2005, p.264). En este sentido, podemos afirmar que la criminalización de la migración supone no solo una "condición de otro" (ESPIRO; ZUBRZYCKI, 2013, p.109) por origen del sujeto, sino que también será atravesado por la discriminación racial y por una "class supremacy" (MILLER, 1928, p.2). En este texto, se incorpora, además, como variable de análisis, el género (MONCLÚS MASO, 2005), que actúa en este caso sobre las masculinidades de estos hombres subalternos (MENJIVAR OCHOA, 2017). Se entenderá entonces que la criminalización de los senegaleses se debe a su condición de extranjero, a su condición de clase, a su condición racial y de género. No obstante, la articulación de las categorías que se propone para analizar la situación de la comunidad senegalesa es la interseccionalidad (CRENSHAW, 2011). Aunque Kimberle Crenshaw incorporó esta definición en el año 1989 en el texto Demarginalizing the intersection of race and sex, el feminismo negro impulsó esta mirada multidimensional sobre la discriminación de la mujer negra desde muchos años antes (VIVEROS VIGOYA, 2016). La característica de la interseccionalidad es que no propone una sumatoria de discriminaciones, sino que advierte que diferentes discriminaciones pueden intersectar en una complejidad diferente que no es solo la suma de las partes, 
sino una forma de discriminación distinta, interseccional (CRENSHAW, 1991). Lo novedoso del texto del año 1989 de Crenshaw es que incorpora esta palabra para dar cuenta de la discriminación en la justicia, analiza casos de trabajadoras contra la multinacional General Motors y como, incluso la mirada de múltiples discriminaciones no da cuenta del fenómeno de la interseccionalidad:

La negativa de la corte en DeGraffenreid a reconocer que las mujeres negras enfrentan discriminación combinada con la raza y el sexo implica que los límites de la doctrina del sexo y la discriminación racial están definidos respectivamente por las experiencias de las mujeres blancas y de los hombres negros. Bajo este punto de vista, las mujeres negras están protegidas solo en la medida en que sus experiencias coincidan con las de cualquiera de los dos grupos (CRENSHAW, 1989, p.143, traducción propia).

Un aspecto importante que se desprende de lo señalado es el concepto de masculinidades analizado desde la interseccionalidad. Este aspecto fue enormemente abordado desde una mirada crítica (KIMMEL, 1992), siendo el máximo exponente Viveros Vigoya (2016), quien trabajó sobre la particularidad del hombre negro e indígena en América Latina. En este texto se recuperan estos análisis para comprender la comunidad senegalesa migrante ya que, pese a que está documentada la existencia de migrantes mujeres (ZUBRZYCKI, 2016), la mayoría de los senegaleses en el país son hombres jóvenes de 18 a 35 años (KLEIDERMACHER, 2012). Estos hombres subalternos, no hegemónicos, soportan una inusitada violencia institucional bajo la justificación de que son peligrosos para el Estado, asociando al hombre negro con el crimen. Este proceso de deshumanización transforma a estos hombres subalternos en sujetos espectrales. Esta negación de humanidad los convierte en sujetos pasibles de recibir violencia.

Otro aspecto importante que merece una aclaración es la cuestión racial. En este trabajo se entiende que "Raza es signo, y su único valor sociológico radica en su capacidad de significar. Por lo tanto, su sentido depende de una atribución, de una lectura socialmente compartida y de un contexto histórico y geográficamente delimitado" (SEGATO, 2007, p.76). Para delimitar específicamente que aspectos se consideran relevantes en este trabajo, es necesario dar cuenta mínimamente del racismo estructural en el país, para poder señalar después, porque se considera que en el período de gobierno de la Alianza Cambiemos la persecución racial fue en aumento y se volvió sistemática contra los senegaleses. 
En este sentido es importante señalar que, desde la propia concepción del Estado argentino, las elites manifestaban la necesidad de "blanquear" a la población argentina para reducir el carácter mestizo de la misma. El caso más emblemático por su peso en la historia nacional es Domingo Faustino Sarmiento, pero no es el único, Alberdi, "padre" de la constitución nacional se expresaba en los mismos términos (ALBERDI, 1916). Estos argumentos terminaron haciendo parte del propio texto constitucional de 1853, que hasta el día de hoy sigue vigente como la carta magna del Estado argentino:

El Gobierno federal fomentará la inmigración europea; y no podrá restringir, limitar ni gravar con impuesto alguno la entrada en el territorio argentino de los extranjeros que traigan por objeto labrar la tierra, mejorar la industria, e introducir y enseñar las ciencias y las artes (ARGENTINA, 1994, art.25).

Esta mezcla que se proponía tenía como objetivo la invisibilización de la mayoría de la población negra e indígena (GARCÍA, 2010) con el paso de las generaciones. Esta activa política de blanqueamiento se completó con la negación de la existencia negra e indígena en estadísticas (LYMAN, 1979), en la historiografía (RÍO ORTIZ, 2009) y desde la simbología nacional (CIRIO, 2003). El objetivo era la negación e invisibilización total. Como contrapartida, al negar la negritud local, se refuerza lo que García (2010, p.12) denomina “extranjerización”, es decir, todo no blanco es extranjero. En este sentido opera el racismo frente a la presencia de los migrantes senegaleses, más aún en la Ciudad de Buenos Aires, que, a diferencia del resto del país, tiene una población que es en su mayoría fenotípicamente blanca. La extranjerización, entonces, en este contexto se presenta como hipervisibilización (GARCÍA, 2010), son hipervisibles en las calles de una ciudad que se auto reconoce como blanca.

\section{La comunidad senegalesa en el país}

Interesa en este apartado hacer una breve reseña sobre la comunidad senegalesa en la Ciudad de Buenos Aires. Se presentan algunas características de la misma como la cantidad de migrantes, su inserción laboral y los problemas habitacionales. Cabe señalar que el hecho de que la mayoría de los migrantes senegaleses se encuentren en situación documental irregular los afecta notoriamente tanto en el trayecto migratorio transnacional, como en su inserción en el país de destino y la posibilidad de ejercer sus derechos allí. La migración senegalesa en Argentina 
es una migración reciente, ya que, pesar que se cuentan con registros de ingresos al país desde los años 90, recién en la primera década del siglo XXI, se consolidó como la comunidad africana más grande de Argentina (ZUBRZYCKI, 2009). El último censo realizado en el año 2010, arrojó un registro de 459 senegaleses en el país de los cuales el 54,7\% vivían en la Ciudad de Buenos Aires. Las investigaciones realizadas sobre este resultado dan cuenta de que este número es un subregistro (KLEIDERMACHER, 2016). De hecho, ya para el año 2010 la Asociación de Residentes Senegaleses en Argentina (ARSA) señalaba que la comunidad en el país estaba compuesta por 2500 migrantes aproximadamente (KLEIDERMACHER, 2016). Más allá del subregistro, la distribución de los migrantes en el territorio continua el mismo patrón del censo, es decir, la mayoría vive y trabaja en la Ciudad Autónoma de Buenos Aires (ZUBRZYCKI, 2016). Para el año 2015, los referentes de ARSA señalaban que había aproximadamente 5000 migrantes senegaleses en Argentina (PACECCA; CANELO; BELCIC, 2017). Número que se mantiene estable, según afirman, desde ese año (PÉREZ RABASA, 2019). Este dato, ARSA lo construye calculando la cantidad de concurrentes a la celebración del Gran $\mathrm{Magal}^{3}$ que realizan cada año. El último evento, se realizó en el estadio de All Boys, en la Ciudad de Buenos Aires. Pero también se realizaron festejos más pequeños en Córdoba y en La Plata (PÉREZ RABASA, 2019). El carácter irregular de esta migración, y la característica transnacional del viaje a la Argentina desde Senegal, hacen que no haya registros oficiales confiables y que sea difícil saber con exactitud cuántos migrantes se encuentran en el territorio. No obstante, el dato sobre la cantidad de senegaleses que proporciona ARSA es coincidente con las investigaciones producidas en Ecuador y Brasil, ambos países "llaves" de ingreso de la comunidad al continente. Durante la primera década del siglo XXI, el ingreso mayoritario de los senegaleses al país se hacía a través de Brasil (TEDESCO; GRZYBOVSKI, 2011). Tramitar una visa turística a Brasil desde la capital de Senegal ${ }^{4}$ permitía en principio tomar un vuelo hacia el continente. Esta "llave" a América permitía luego la llegada al país de destino por una frontera terrestre (ZUBRZYCKI, 2013b). Luego de esta primera década, Brasil "endureció” el otorgamiento de visados para esta comunidad (GOLDBERG; SOW, 2017). Cuando Brasil se comenzó a presentar problemático como estrategia de entrada al continente, Ecuador fue la alternativa, sobre todo desde el año 2008, cuando Ecuador aprobó la nueva constitución que señalaba “(Ecuador) Propugna el principio de ciudadanía universal, la libre movilidad de todos los habitantes del planeta y el progresivo fin de la condición

\footnotetext{
3 Esta es la celebración anual más importante de los senegaleses pertenecientes a la cofradía Mouride de origen Wolof que son mayoritarios dentro de la comunidad migrante en Argentina (KLEIDERMACHER, 2015).

4 Brasil cuenta con una embajada en la ciudad de Dakar. Argentina no.
} 
de extranjero como elemento transformador de las relaciones desiguales entre los países, especialmente Norte-Sur" (ECUADOR, 2008, Art 416, Inc. 6). Esta nueva legislación eximía a los viajantes del pedido de visado, de esta forma el ingreso al continente era más sencillo, pero el país de destino se presentaba un poco más lejos, y el recorrido por tierra hasta la frontera más complejo. Según refiere el Instituto Nacional de Estadísticas y Censos de Ecuador, esto se ve reflejado estadísticamente sobre todo desde el año 2012, donde pasan a ser 247 los ingresos de senegaleses al país contra 22 del año anterior. Otro dato interesante es que los egresos registrados el año 2011 son 21, uno menos que los ingresos; para el año 2012, los egresos informados son solo 62, esta tendencia crece hasta el año 2015 con 3473 ingresos y 71 egresos solamente (INEC, 2019). A finales del año 2015, el Estado ecuatoriano anunció que pediría visado a los ciudadanos provenientes de Senegal (Acuerdo Ministerial $N^{\circ}$ 088, ECUADOR, 2015), para el año 2016, el número de ingresos y egresos volvió a ser parecido a lo que era previo al 2012, 61 ingresos y la misma cantidad de egresos (INEC, 2019). Se puede afirmar sobre los datos producidos por el Instituto Nacional de Estadísticas y Censos de Ecuador que aproximadamente 6 mil senegaleses permanecieron en el continente (MÉNARD MARLEAU, 2017). Por otra parte, el registro de ACNUR (2014) para Brasil da cuenta de una tendencia creciente de solicitudes de refugio de senegaleses en ese país desde el año 2012, siendo 161 solicitudes ese año y 1687 en el año 2014. En el caso argentino, la tendencia creciente de solicitudes de refugio se puede observar desde el año 2006 aproximadamente (PÉREZ RABASA, 2019). Al solicitar el estatus de refugiado, lo que obtienen los migrantes es un certificado provisorio, conocido como "precaria", este documento es válido en tanto se resuelva la situación en la Comisión Nacional de Refugiados, un trámite que puede llevar bastante tiempo. Mientras esto suceda, se encuentran provisto de algún tipo de documentación (ZUBRZYCKI, 2013a). Estas estrategias también implementaron como comunidad en Brasil (MÉNARD MARLEAU, 2017), no obstante, a diferencia de Brasil, en Argentina hubo una disposición especial de regularización de migrantes senegaleses (Disposición N² del 2013), que habilitó estrategias de regularización diferentes por parte de la comunidad. Es importante señalar que la disposición de regularización fue conflictiva en su aplicación, y solo logró otorgar documentos permanentes ${ }^{5}$ a 1191 (ARGENTINA, 2013) ${ }^{6}$. La mayoría de los que se acogió al plan no pudo termi-

\footnotetext{
El Estado otorga un documento transitorio llamado "precaria" mientras el migrante realiza el trámite de regularización, luego de concluido el mismo otorga un documento de "residencia temporaria" y luego de dos años, se puede realizar el trámite para obtener un documento de "residencia permanente". Este último es el documento definitivo de los migrantes en Argentina.

6 Esta información se obtuvo tras un pedido de información pública realizado por "Red Acción", y la correspondiente solicitud de la misma para incluirla en este trabajo.
} 
narlo (PÉREZ RABASA, 2019). Sin embargo, no se desarrolla esta cuestión por no ser materia de análisis del presente trabajo. En cuanto a la irregularidad migratoria, es importante señalar que la legislación migratoria de Argentina (ARGENTINA, 2003) marca una distinción entre migrantes MERCOSUR, que pueden acceder a la regularización por el solo hecho de migrar desde países integrantes o asociados al mismo, e integrantes extra-MERCOSUR, que deben dar cuenta de algunas de las disposiciones que se encuentran en el artículo 23 para poder acceder a la regularización. Además, los ingresos al país por pasos irregulares, por las características de la movilidad transnacional, los imposibilita en el futuro para la realización de este trámite en la Dirección Nacional de Migraciones. Respecto de la solicitud de refugio, aunque otorga una documentación transitoria mientras se realiza el trámite, en líneas generales, la Comisión Nacional de Refugiados no considera a los migrantes senegaleses como refugiados y revota sistemáticamente las solicitudes. Esto se debe a que los considera migrantes económicos, es decir, personas que no se ven forzadas a abandonar su país por persecución racial, religiosa, de nacionalidad o por pertenencia a un determinado grupo social, ni que su vida, su libertad o su seguridad corra peligro, ni exista en Senegal un conflicto armado o violaciones masivas de derechos humanos. El pedido de refugio por parte de la comunidad es simplemente una estrategia para regularizar su situación documental. Si se toma el periodo de 1985 al 2016, que es el último año del cual se disponen datos, de 3223 solicitudes de refugio por parte de senegaleses solo se han otorgado 83, aproximadamente el 3\% de los solicitantes obtuvieron el refugio (PÉREZ RABASA, 2019).

Así que, aunque no se cuentan con registros oficiales, se puede señalar tras analizar las investigaciones producidas tanto en Argentina como en Brasil (MÉNARD MARLEAU, 2017), que la comunidad senegalesa en el período analizado, 2015-2019, se mantuvo estable en cuanto al número, siendo aproximadamente 5 mil personas las que la componen. Este número, aunque es una estimación, permite afirmar que la comunidad senegalesa es muy pequeña en relación a otras comunidades en el país, apenas el 0,27\% del total de migrantes en el territorio. Este porcentaje tan pequeño choca con el hecho de que el Estado no logre dar una respuesta efectiva de regularización. Como la mayoría de los migrantes senegaleses en Argentina se asientan en la Ciudad de Buenos Aires y desarrolla su actividad laboral allí, es importante analizar específicamente como es la organización de los migrantes en este territorio, en que barrios se asientan y en que barrios desarrollan su actividad. Es destacable, también, que el trabajo como la vivienda son cuestiones críticas en la Ciudad más allá de lo que le sucede a esta comunidad (TREVISANI VESPA; MAMBERTI, 2012), no obstante, el carácter irregular de la migración los expone a situaciones de mar- 
ginación tanto en lo laboral como en lo que respecta a la vivienda. En cuanto a la vivienda, predomina la estrategia de agrupamiento. Esto por un lado permite abaratar los costos de alquiler y, por otro, sortea un problema generalizado en la comunidad que es la falta de documentación, cuestión necesaria para conseguir un arriendo. Como las posibilidades de alquiler son limitadas, se hacen en condiciones precarias, mayoritariamente en pensiones que se encuentran emplazados en los barrios de Balvanera, Constitución, Flores, Liniers, Barracas, Parque Patricios, Nueva Pompeya fundamentalmente, pero también en otros puntos de la Ciudad. Además, funcionan como hogar los hoteles inhabilitados por el Gobierno de la Ciudad. Los dueños de estos alquilan piezas a familias de migrantes, en Balvanera, Constitución, Flores y Liniers sobre todo, pero no solamente en esos barrios (BUENOS AIRES, 2018a). Entre las estrategias menos populares en la comunidad senegalesa encontramos dos, la estrategia del subalquiler, es decir, un migrante alquila un departamento y luego subalquila a compatriotas. Finalmente, la vivienda compartida en los asentamientos de la Ciudad, en los que se encuentran en el Sur, Villa Zabaleta, 1-11-14, y los que no, como en la Villa 31. Las condiciones de estas viviendas son generalmente malas y la necesidad de agruparse para costear las mismas genera hacinamiento, dos factores de notable impacto en la comunidad (KLEIDERMACHER, 2017). No obstante, esta estrategia abarata costos, ya que no es solo la vivienda, sino que también se comparten las comidas. Además, vivir juntos otorga una red de contención para cualquier necesidad que se tenga (RUCHANSKY, 2009), incluso ante el robo de mercadería por parte de las fuerzas de seguridad, lo que permite conseguir con facilidad nuevamente algo para vender (KLEIDERMACHER, 2012). En cuanto a la actividad laboral, la mayoría de los senegaleses en el país se desempeña como vendedor ambulante. Esta actividad de carácter móvil les da la posibilidad de traslado para buscar mayores ganancias dependiendo las temporadas de ventas. Por otro lado, es una actividad que en otras latitudes también desempeńa la comunidad migrante senegalesa (LACOMBA VÁZQUEZ, 2018). Otro factor que incide en la elección de esta actividad es el idioma, ya que no se requieren mayores conocimientos del español para desempeñarla, con solo aprender algunas palabras, un senegalés recién llegado puede empezar a trabajar (SARUBBI, 2014). Finalmente, se afirma que el hecho de que la propia comunidad facilite la mercadería apenas el migrante llega al país y algún compatriota lo acompañe en los primeros días, mostrando donde puede trabajar y cómo hacerlo, resulta fundamental para los recién llegados (KLEIDERMACHER, 2012). Estas "complejas redes sociales, comerciales y religiosas" (REITER, 2010, p.20) funcionan como sostén cuando las fuerzas de seguridad les decomisan la mercadería. La 
posibilidad de endeudarse con un compatriota para poder volver a vender es una estrategia extendida en la comunidad, aunque no debe entenderse solo como una red comercial, el aspecto económico es sin dudas importante. Otra estrategia de obtención de la mercadería para algunos senegaleses es la compra de productos por cuenta propia en el barrio de Once fundamentalmente. Consiguen mercadería para revender en los mayoristas emplazados en este barrio, pero también existen "senegaleses mayoristas", que compran en grandes cantidades y reparten mercadería por los hoteles, concesionada o no, dependiendo el caso (ZUBRYCKI, 2016). Todos estos elementos son centrales para entender el proceso de criminalización al que fueron sometidos por el Estado en este período, no obstante, es necesario señalar que muchas de las situaciones de vulneración de derechos que se vivieron en este período tienen una raíz mucho más profunda, que trasciende la gestión liberal-conservadora de la Alianza Cambiemos, aunque en este período se puede constatar ciertas características particulares que se señalan en el siguiente apartado.

\section{Criminalización interseccional}

Durante el período que se analiza, hubo un giro en la política migratoria que significó un retroceso de los derechos de todos los migrantes:

Las distintas medidas adoptadas por el Gobierno de la alianza Cambiemos, desde su llegada al poder en 2015, se basaron en una vinculación directa entre migración y criminalidad, dirigida a reinscribir la política migratoria en el terreno de la seguridad y a enfatizar el control, en detrimento de la integración de las personas migrantes (PENCHASZADEH; GARCÍA, 2018, p.91).

Lo notable del caso es que este período caracterizado por un retroceso en materia de derechos humanos de migrantes también fue atravesado por una serie de informes para evaluar la situación de los derechos humanos en el país, por parte de Naciones Unidas (ONU), en lo que se puede llamarse la "segunda ronda de informes en la historia de la Argentina ante los Comités de Naciones Unidas" (JARAMILLO FONNEGRA, 2019, p.73). Con esto se señala que no solo significó la asunción de la Alianza Cambiemos un cambio en los discursos prácticas y leyes en relación a migración, sino que además existieron una serie de reclamos de distintos órganos del Sistema Internacional de Derechos Humanos que dieron cuenta de esta situación. La vinculación de la migración con el delito 
se evidencia en el discurso presidencial de inauguración de las sesiones ordinarias en el Congreso de la nación en marzo del 2016 (DISCURSO..., 2016) donde el entonces presidente vinculó a los migrantes con el narcotráfico; también en el aumento de expulsiones durante los cuatro años de gestión (DURANTE..., 2019) y en el Decreto de Necesidad y Urgencia 70 del año 2017 (PÉREZ RABASA, 2018), que se desarrolla brevemente en el siguiente apartado, por solo mencionar tres elementos. Estos cambios en la política migratoria han afectado a toda la comunidad migrante en el país, no obstante, en este apartado solo se hace referencia a los cambios que han impactado notablemente a la comunidad senegalesa en CABA.

\subsection{Normativa excluyente}

La ley de migraciones $\mathrm{N}^{\circ} 25.871$, fue sancionada el 17 de diciembre del año 2003. Tal como se señaló más arriba, a pesar de ser una legislación que incorporaba estándares de derechos de migrantes, y que fue notablemente elogiada por los órganos del Sistema Internacional de Derechos Humanos (CERIANI CERNADAS, 2004), la ley planteó una división entre migrantes de MERCOSUR y de extra-MERCOSUR, en este sentido, le daba reconocimiento a los migrantes limítrofes que eran en ese momento, y son ahora todavía, los migrantes más numerosos en el país. No obstante, los migrantes que no pertenecen a esta comunidad de naciones, como es el caso de los senegaleses, no encuentran una "ventana" de regularización, ya que no pueden dar constancia de su ingreso al país, por haberlo hecho de forma irregular, no pueden dar cuenta de un trabajo y acogerse a la figura del "trabajador migratorio", porque la ley no reconoce la venta ambulante como tal, y por otro lado, tal como se detalló, el Estado tampoco los reconoce como refugiados, con lo cual la irregularidad documental es el destino para la mayoría de la comunidad senegalesa. Por esta razón es que, tras presiones de la propia comunidad senegalesa, la Dirección Nacional de Migraciones decidió realizar un régimen especial de regularización en 2013, que permitió al 20\% de la comunidad, aproximadamente, obtener un documento definitivo. A estos señalamientos, que se refieren a la situación migratoria de esta comunidad previo al año 2016, hay que sumar el hecho de que con la asunción de la Alianza Cambiemos, se modificó por decreto la ley de migraciones (ARGENTINA, 2017), cuestión que puso a los migrantes senegaleses en una situación de vulneración mucho mayor a la ya mencionada irregularidad. El Decreto de Necesidad y Urgencia 70 del 2017 sustituye diez artículos de la Ley de Migraciones, incorpora quince, y deroga uno. Pero son 
las modificaciones de los artículos 29, el 54 y el 86 las más relevantes para este tema, por los cambios en los plazos y las formas respecto de la asistencia jurídica gratuita, la notificación y el recurso de revisión, tanto como la asociación de la irregularidad a la criminalidad. El artículo 29 se refiere a las causales impedientes de ingreso y permanencia del país, enumerando una serie de cuestiones como por ejemplo "Tener antecedentes por actividades terroristas", "Haber incurrido o participado en actos de gobierno o de otro tipo, que constituyan genocidio, crímenes de guerra, actos de terrorismo o delitos de lesa humanidad", entre algunos otros. El inciso más problemático para la comunidad senegalesa es el K que refiere "Intentar ingresar o haber ingresado al territorio nacional eludiendo los controles migratorios, o por lugar o en horario no habilitados al efecto" (inc. k). Esta cuestión ya estaba presente en el texto de la ley original, y nos permite señalar que equiparar un acto terrorista con la irregularidad migratoria es sin dudas una forma de criminalización de la migración más vulnerable. No obstante, con la modificación del Decreto al inciso c del artículo 29, se elimina la limitación temporal de las penas "Haber sido condenado o estar cumpliendo condena, o tener antecedentes o condena no firme en la República Argentina o en el exterior, por delitos que merezcan según las leyes argentinas penas privativas de libertad" (ARGENTINA, 2017, art.29). Esta supresión del límite temporal de 3 años o más que estaba en la ley original, y la incorporación de la "condena no firme", amplia la posibilidad de expulsar migrantes, perjudicando fundamentalmente a quienes las fuerzas de seguridad persiguen cotidianamente, como es el caso de los migrantes senegaleses por el tipo de actividad económica que realizan, ya que son trabajadores de la economía popular (CORAGGIO, 1989), criminalizados por desarrollar su actividad en la calle. Al respecto la Defensoría del Pueblo de la Ciudad de Buenos Aires afirmó: "En tal sentido, a modo de ejemplo, la venta ambulante que pueda implicar infracción a la ley de marcas, una tentativa de hurto, una protesta o manifestación pública que pueda derivar en una investigación penal, son causales que obstan al ingreso y permanencia" (CARRIL, 2017, p.7). La modificación del artículo 54 de la ley de migraciones, versa sobre notificaciones, y para el caso particular de este análisis resulta de suma importancia, ya que como se señaló, las condiciones de vivienda de la mayoría de la comunidad son precarias. La modificación versa:

Si no constituyese domicilio alguno, o el constituido no existiere, los actos emitidos por la Dirección Nacional de Migraciones se tendrán por notificados de pleno derecho, en el término de dos días hábiles, desde el 
momento de su emisión, quedando los mismos disponibles en la mesa de entradas de la citada Dirección Nacional (ARGENTINA, 2017, art.5).

Esto genera un problema práctico, ya que la mayoría viven en hoteles o pensiones donde conviven varias personas en cada pieza, muchos de esos hoteles están en situación de irregularidad o son precarios (KLEIDERMACHER, 2016), pero para la Dirección Nacional de Migraciones un migrante puede estar notificado de una expulsión, aunque esto nunca haya sucedido efectivamente. Es realmente difícil pensar que un senegalés en esta situación precaria de vivienda, se presentará todas las semanas en mesa de entrada de la institución correspondiente para saber si hay una notificación a su nombre. De todas maneras, tal como queda establecido en la nueva legislación, se los considera notificados, los tiempos corren y se vencen los plazos para recurrir la posible sanción administrativa. Se entiende entonces, que este régimen de notificaciones lesiona derechos y afecta fundamentalmente los migrantes con una situación de vivienda precaria. Finalmente se hace referencia a la modificación del artículo 86, sobre la asistencia jurídica. Interesa a los fines del presente texto la aclaración final del artículo "Cuando no haya sido requerida la asistencia jurídica gratuita o no se acreditará de forma fehaciente la falta de medios económicos, se continuará con las actuaciones administrativas sin más trámite" (ARGENTINA, 2017, art.24). En este caso, se pone en cabeza del migrante la solicitud de forma expresa de una asistencia jurídica gratuita, a diferencia del original de la ley, donde era la Dirección Nacional de Migraciones quién debía informar al migrante sobre su derecho. Esto va en perjuicio para los migrantes que pueden no saber que tienen el derecho a la defensa, más grave aún si nos referimos a migrantes que ni siquiera comprenden bien el idioma, como es el caso de la mayoría de los migrantes senegaleses.

Por otro lado, no se aclara que sería "acreditar de forma fehaciente" la situación económica precaria, si debe ser con un informe social", un "certificado

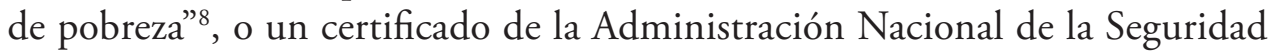
Social $(\mathrm{ANSeS})^{9}$. Al ser difuso este aspecto quedará a criterio de la Dirección Nacional de Migraciones, la aceptación del mismo o el rechazo. Una situación

\footnotetext{
7 Un documento que realiza un Trabajador Social acreditando la situación socio económica de la persona solicitante. Puede solicitarse el mismo, a un organismo público que tenga trabajadores sociales o al propio Colegio de Trabajo Social, en este caso de CABA.

8 Un documento que para el caso de la Ciudad de Buenos Aires otorgan organismos como el Centro de Acceso a la Justicia.

9 Un documento que otorga este organismo y se puede solicitar por internet dando cuenta de la falta de aportes jubilatorios.
} 
inadmisible, teniendo en cuenta que sería el mismo organismo que pide la expulsión quien no brinde la posibilidad de la asistencia jurídica gratuita. Además, no es sencillo, conseguir alguno de estos documentos en los plazos que solicita el Decreto de Necesidad y Urgencia, se expone el ejemplo del "certificado de pobreza" que otorga la Ciudad de Buenos Aires, que se hace con la solicitud de un turno previo que se otorga con un plazo mínimo a una semana y, además, el trámite se hace por internet. Siempre teniendo en cuenta que las modificaciones de la política migratoria perjudican a todos los migrantes, es necesario señalar que el colectivo senegalés es atravesado por una afectación interseccional de sus derechos donde confluyen otros aspectos como la raza. Aunque la vinculación entre racismo y xenofobia se puede rastrear desde el propio acto de fundación de la Argentina, tal como se señaló, existieron cambios específicos que nos permiten decir que la comunidad senegalesa vivió el racismo institucional de forma sistemática en el período del gobierno de liberal-conservador de la Alianza Cambiemos.

\subsection{Persecución racializada}

En este apartado se desarrolla la persecución racializada sobre el colectivo senegalés. La hipervisibilización en las calles de la Ciudad, que es el lugar donde realiza la mayoría su actividad laboral, los afecta a la hora de ser requisados por las fuerzas de seguridad. Ahora bien, la construcción de perfiles raciales no es una característica exclusiva de las fuerzas de seguridad del gobierno de la Alianza Cambiemos, pero el recrudecimiento de la persecución callejera al colectivo senegalés, permite afirmar que existió una criminalización en el período 20152019. Este aumento considerable de la persecución se puede evidenciar en las presentaciones que organizaciones de migrantes han hecho ante el Estado, y en las declaraciones de distintos organismos del Sistema Internacional de Derechos Humanos sobre Argentina en este período.

Se argumenta en este apartado que fue la hipervisibilidad, como persecución racializada, la razón de las detenciones, no la existencia de un delito. En este sentido se pronunció la Confederación de Trabajadores de la Economía Popular ${ }^{10}$ (CTEP) en la Legislatura de la Ciudad el 26 de junio del 2018 sobre un caso de un migrante que fue abordado por las fuerzas de seguridad y detenido, cuando estaba sentado en la vía pública, en su horario de receso laboral. Al respecto, las presidentas de las comisiones de Derechos Humanos y de Prevención de

\footnotetext{
${ }^{10}$ Organización que nuclea vendedores ambulantes, textiles, cartoneros y varias otras actividades que no son reconocidas como trabajo por las organizaciones sindicales tradicionales.
} 
la Violencia Institucional manifestaron "Los agarran por negros, no por estar vendiendo" (EL RECLAMO..., 2018, p.1). En este mismo evento público, un referente de la comunidad senegalesa advirtió: "Desde 2015 hay mucha represión policial. Los chicos están sufriendo mucho acá en Argentina ahora. Los acusan de cualquier cosa" (LA LEGISLATURA..., 2018, p.1). Otro referente de la comunidad senegalesa se refirió a esta cuestión en el Centro de Estudios Legales y Sociales, a pocos días del evento antes mencionado: "En este momento es peor que antes, porque antes trabajábamos más tranquilos, estábamos más tranquilos porque no nos molestaban, pero ahora se cambiaron las leyes, todo cambió y todo es más duro." (CELS, 2018, p.1).

Pero no solo las organizaciones sociales y de migrantes registraron casos de forma sistemática, sino que también existen registros de organismos del Sistema Internacional de Derechos Humanos que dan cuenta de este fenómeno como el Informe del Relator Especial sobre las formas contemporáneas de racismo, discriminación racial, xenofobia y formas conexas de intolerancia que manifestó en su misión a la Argentina en 2017:

El Relator Especial fue informado de una tendencia en la Policía Metropolitana de la Ciudad de Buenos Aires y la Policía Federal Argentina, que se basa en utilizar perfiles en los controles de identidad que se realizan en las calles. Esta práctica afecta desproporcionadamente a los migrantes y a los afrodescendientes. Aparentemente, el uso de perfiles raciales ha aumentado tras una decisión adoptada en 2015 por el Tribunal Superior de Justicia de la Ciudad Autónoma de Buenos Aires, de revocar una sentencia que había declarado nulas las detenciones vinculadas a los controles de identidad, argumentando en la decisión que la Policía Federal podía detener aleatoriamente a las personas para realizar controles de identidad sin que existiera la sospecha de la comisión de algún delito. (NACIONES UNIDAS, 2017, p.73).

Tal como señaló el relator, la persecución en la calle tiene un aval judicial, que permite a la policía detener sin existencia de delito, pero, sin dudas, esta modificación del año 2015 no afectó a todos de la misma manera, siendo el factor racial fundamental. Así lo entendió el Grupo de Trabajo de Expertos de las Naciones Unidas sobre Afrodescendientes, que manifestó en su visita a la Argentina en marzo del 2019 "Debe ponerse fin al excesivo control policial de los/as vendedores/as ambulantes de origen africano (...)” (NACIONES UNIDAS, 2019, p.48). 
Otro factor notable, que da cuenta de la sistematicidad de la persecución, son los operativos en conjunto de las fuerzas de seguridad con el Ministerio de Ambiente y Espacio Público contra senegaleses, que llamaremos "megaoperativos" (PÉREZ RABASA, 2019), por el enorme despliegue de personal que suponen. Así que a los pequeños operativos diarios que se señalaban antes, hay que sumar que a partir del ańo 2016 los "megaoperativos" se volvieron sistemáticos en los distintos barrios de la Ciudad, siendo los primeros en Liniers y en Flores en el 2016 (FUERTES..., 2016), en Constitución y en Once en el 2017 (MIEMBROS..., 2017), en Liniers nuevamente en el 2018 (TENSIÓN..., 2018) y finalmente Flores y Once ese mismo año (MEGA..., 2018). A diferencia de las detenciones cotidianas, los "megaoperativos" contaban con una enorme cobertura mediática, presentando a los migrantes senegaleses como parte de una mafia internacional de la venta ambulante, mientras las imágenes mostraban como eran desalojados de su vivienda y despojados de su mercadería.

La persecución callejera, tiene por otro lado, una contracara, que es la estadística de detención. Los registros de las organizaciones sociales, académicas y de derechos humanos sobre detenciones es la única fuente con la que se cuenta (PÉREZ RABASA, 2019), ya que el Ministerio de Justicia de la Nación y de la Ciudad sistemáticamente negaron el acceso a tales datos. Sobre esta cuestión se expidió el Comité para la Eliminación de la Discriminación Racial [CERD], que, en sus observaciones finales del informe para la Argentina de Diciembre del 2016, advirtió:

El Comité lamenta la falta de información estadística desglosada sobre las denuncias por discriminación racial y las correspondientes actuaciones. (...) Preocupa también al Comité la falta de un trato equitativo en el acceso a la justicia cuando se trata de población afrodescendiente (CERD, 2016).

Es destacable que el CERD se refiera a la falta de estadísticas y al trato equitativo en el acceso a la justicia en el mismo párrafo, ya que la invisibilización estadística deriva en una negación del problema. Si no se visualiza la problemática, no se puede abordar para transformarla.

En este apartado no se pretendió sistematizar todos los casos, sino solo exponer algunos ejemplos que dan cuenta de la construcción de un perfil racial en las detenciones, y como estas se volvieron sistemáticas. Sobre lo que no se hizo referencia es a la violencia en los operativos de las fuerzas de seguridad, cuestión que se entiende, se relaciona con la forma en que el Estado actúa sobre 
estas "masculinidades disminuidas de los conquistados" (MENJIVAR OCHOA, 2017, p.364).

\subsection{Abordaje violento}

El hecho de que la mayoría de los migrantes senegaleses en el país sean hombres es un dato significativo que vincula al género con la clase, por el carácter proveedor de esta. Pero este asunto no debe entenderse exclusivamente desde un aspecto económico, pues estas masculinidades se transforman en el proceso de la migración, tal cual detallan varios trabajos académicos realizados sobre senegaleses en la Argentina:

El emigrado se percibe en origen como un exitoso, un referente social: hacerse hombre, tener dinero, mujer e hijos. El prestigio se manifiesta no sólo en términos materiales sino sobre todo sociales y simbólicos, ya que el emigrante exitoso es aquel que mantiene la responsabilidad moral de redistribuir su riqueza, manteniendo financieramente a su familia (ZUBRZYCKI, 2009, p.5).

Otro aspecto destacable en ese sentido es la expresión Modou-Modou, vocablo utilizado en Senegal para designar a los migrantes hombres de la etnia Wolof, que envían remesas a sus familias (SOW, 2004). Esta no es una designación hacia todos los migrantes, sino hacia los hombres migrantes, un aspecto que revela la masculinización de esta migración en todo el mundo, no solo en Argentina, además, el carácter histórico de la migración masculina de Senegal. La contracara de la migración masculina es el rol de las mujeres en el país de origen como "jefas" del hogar. Estos roles definidos deben entenderse a la luz de la cultura senegalesa fuertemente atravesada por la religión musulmana (ZUBRZYCKI, 2009).

Por otro lado, el enfoque de género vinculado al racismo permite explicar la violencia que ejerce el Estado contra estas "masculinidades disminuidas" (MENJIVAR OCHOA, 2017, p.364). La idea del hombre no-blanco como un ser salvaje, animalesco, se presenta como una justificación de la acción estatal violenta. Al representar seres no civilizados que son "vulnerables a la ejemplaridad de la masculinidad victoriosa” (SEGATO, 2016, p.19). La construcción de una imagen racista del hombre negro salvaje en contraposición con el blanco civilizado funciona como una justificación subyacente para ejercer violencia sobre estos cuerpos. 
Lógicamente, esta cuestión afecta a todas las masculinidades racializadas y no solo a los senegaleses, tal como señala el Centro de Estudios Legales y Sociales en su informe anual sobre Argentina "El foco principal del hostigamiento son los jóvenes varones pobres” (CELS, 2016, p.13). No obstante, la inusitada violencia policial sobre este colectivo migrante compuesto mayoritariamente por jóvenes, pobres y racializados se puede entender desde el mensaje que el gobierno de la Alianza Cambiemos pretendió dar. Tal cual define Segato (2016, p.39) "Es por su calidad de violencia expresiva más que instrumental — violencia cuya finalidad es la expresión del control absoluto de una voluntad sobre otra”. La violencia que se ejerce sobre un migrante senegalés es a la vez una forma de opresión al sujeto en sí, pero también un mensaje para el resto. Sobre este accionar violento y sistemático se expresó el informe anual del Programa de Violencia Institucional del Ministerio Público de la Defensa:

Según los casos arrimados al Programa contra la Violencia Institucional, se ha detectado que la operatoria realizada por las fuerzas de seguridad consiste en que se les aproxima en la calle, y sin mediar pregunta o requerimiento alguno, le sacan sus pertenencias utilizando la fuerza y cuando, ante esta situación de violencia, el vendedor intenta solicitar alguna explicación, la policía lo inmoviliza, aumentando la violencia ejercida, y el operativo termina con el vendedor detenido (BUENOS AIRES, 2017a, p.6).

Al respecto, también se refirió el Grupo de Trabajo de Expertos de la Naciones Unidas sobre Afrodescendientes en su visita a la Argentina: "El uso de la fuerza en forma desproporcionada contra los afrodescendientes puede resultar en una violencia letal" (NACIONES UNIDAS, 2019, p.21). Con este sistemático accionar, los migrantes senegaleses desarrollan su vida en el país con miedo constante a la violencia policial. Más aún, si se tiene en cuenta la enorme exposición que tienen por el desarrollo de su actividad laboral.

\subsection{Sobre el trabajo}

El primer aspecto importante para destacar respecto de la clase es que los migrantes que viajan hacia el país no son los senegaleses más pobres, ya que viajar implica en principio un vuelo hasta el continente, y tal como se explicó, un viaje transnacional que puede significar más de un país. Todo este corredor migratorio implica dinero, por eso los migrantes que llegan a la Argentina venden negocios para viajar, ahorran mucho tiempo, o las familias costean el 
viaje de alguna forma (KLEIDERMACHER, 2016). En este proceso, estos migrantes se desclasan, descubriéndose trabajadores de la economía popular (CORAGGIO, 1989) en el país de destino y cualquier jerarquía de clase en el país de origen se desdibuja para igualarlos en el espejo que les presenta el país de destino de la emigración

Estos migrantes desclasados que desarrollan su actividad laboral en la calle son perseguidos por las fuerzas de seguridad, tal como se señaló en el apartado anterior, y son sancionados judicialmente bajo la presunción de la violación a la Ley de Marcas y Designaciones ( $\mathrm{N}^{\circ}$ 22.362). La utilización de esta normativa para sancionar a los senegaleses que venden bijouteri o ropa deportiva pareciera desproporcionada, si tenemos en cuenta que los supuestos damnificados son empresas multinacionales con un poder de lobby político y económico enorme. Según indica la Cámara Argentina de Comercio y Servicios en sus Informes de Venta Ilegal Callejera y Piratería en la Ciudad de Buenos Aires, desde hace varios años las marcas falsificadas que se venden mayoritariamente en la calle son Nike, Adidas, Puma, Lacoste, Ray Ban, Rolex e Infinit (CAC, 2019).

Estas multinacionales han encontrado en desde el año 2015, un interlocutor afable en el Estado con su concepción de la venta ambulante. Esto se evidencia en las reuniones públicas entre representantes de ambas partes, multinacionales y Estado, elogiándose por la política hacia los migrantes senegaleses. La primera fue con el Director Mundial de Protección de Marca, de la empresa Levi Strauss en el 2016. El martes 6 de junio se realizó un acto en la sede de la fiscalía penal, contravencional y de faltas $\mathrm{N}^{\circ} 35$, fiscalía que lleva prácticamente todas las causas de senegaleses en la Ciudad. Al año siguiente, en el 2017, la empresa Nike premió a la fiscalía mencionada, y al Subcomisario Sergio Herrera, del área de contravenciones de la policía de la ciudad. El evento tuvo lugar en la sede administrativa de la multinacional en un ágape donde participaron los máximos representantes de la empresa (BUENOS AIRES, 2017b).

En el 2018, fueron varias las empresas que presentaron públicamente su conformidad con las políticas hacia los vendedores ambulantes. El 6 de agosto del 2018 el Ministro de Justicia y Seguridad de la Ciudad recibió a representantes de las empresas Puma, Tommy Hilfiger, Nike, Chanel y Novartis. Estas lo premiaron y lo elogiaron, según manifestó el ministro, por su política contra los vendedores ambulantes, a los cuales denominó en esa reunión como "esta gente” (BUENOS AIRES, 2018b). En el 2019, la empresa Chanel, el miércoles 6 de febrero se dio cita con los funcionarios de la fiscalía $N^{\circ} 35$ en la embajada de Francia. No solo estaba un alto representante de la empresa a nivel mundial, sino también el embajador de Francia, varios funcionarios públicos y funcionarios 
de la Gendarmería Nacional y la Policía de la Ciudad, ambas fuerzas recibieron distinciones al igual que los fiscales (BUENOS AIRES, 2019).

Como se evidencia, las multinacionales se muestran en estas reuniones conformes con la política contra los vendedores ambulantes. Es importante recordar que para el año 2016 ya existían advertencias de organismos internacionales sobre la violencia contra los senegaleses por parte de las fuerzas de seguridad. Aun así, las reuniones y presentaciones públicas dan cuenta del apoyo al gobierno en este tipo de prácticas, las cuales son calificadas por las empresas multinacionales como "un ejemplo a seguir" (BUENOS AIRES, 2018b).

\section{Conclusiones}

Durante el presente trabajo se propuso analizar la criminalización de los senegaleses desde una perspectiva interseccional. En este sentido, no debe entenderse los diferentes aspectos analizados de forma separada, por el contrario, estas afectaciones intersectan construyendo un "ellos" criminal que debe ser entendido en su integralidad. Por ello, se expone a continuación un análisis interseccional, para dar cuenta de la complejidad del fenómeno.

La migración transcontinental que realizan estos migrantes, implica moverse por al menos un tercer país, en el mejor de los casos, y en no pocas ocasiones implica cruzar más países. La inseguridad de este corredor migratorio es alta y pierden buena cantidad de recursos económicos en este camino hacia el sur del continente americano. Una vez en el país de destino, el cruce irregular por pasos fronterizos no habilitados les genera un perjuicio a la hora de intentar regularizarse.

La incorporación en la economía popular, ejerciendo la venta en la calle tiene que ver, por un lado, con un desclasamiento que se produce en la migración $y$, por otro, por la posibilidad de acceder a la mercadería y a un lugar en la calle al día siguiente de haber arribado al país. Esta actividad les hace pasar muchas horas en la calle, expuestos a las fuerzas de seguridad. La disputa por el territorio no es solo con el colectivo senegalés, pero si los afecta particularmente por el racismo estructural y su expresión institucional que se conjugaron como una trampa para un colectivo que, no solo no logra regularizarse en los términos de la actual ley, sino, que la violencia del Estado los convierte en "no-regularizables" en un futuro, por las causas judiciales que pesan sobre la mayoría.

Ante la imposibilidad de desarrollar libremente la actividad laboral, los migrantes buscan estrategias comunitarias para evitar ser abordados por las fuerzas de seguridad. La necesidad de generar dinero para sobrevivir y enviar remesas, 
aunque sean mínimas, los incentiva en estas acciones. No tienen oportunidades de inserción en otra actividad económica, por un lado, por el idioma que se presenta como una barrera y por otro por el racismo estructural que los margina y afecta su derecho a un trato igualitario. El aspecto laboral también se debe leer en esta clave, es muy complejo para un migrante senegalés cambiar de actividad porque no hay lugar en la formalidad laboral para los hombres racializados. Para el caso de los senegaleses que se desempeñan en la venta ambulante, la posibilidad de verse afectados por una causa como, por ejemplo, una infracción a la ley de marcas, es sin dudas alta. En los términos del artículo 29 del Decreto de Necesidad y Urgencia 70, una condena, aunque no esté firme, puede traducirse en una expulsión, o como mínimo, en un obstáculo para regularizarse ante una nueva amnistía como la del año 2013. La construcción de un perfil racial en las detenciones queda en evidencia en el abordaje indiscriminado hacia estos migrantes racializados, estén o no trabajando, estén o no vendiendo de forma ambulante, e incluso en sus propias viviendas. Además, la violencia ejercida en las detenciones contra estos hombres racializados subalternos expone el racismo institucional.

Por otra parte, se puede afirmar que, su desclasamiento en la migración y la legislación migratoria vigente los invita a desarrollar una actividad laboral dentro de la economía popular. Esta actividad los expone ante el Estado municipal de la Ciudad que los persigue por intentar subsistir, privilegiando a las multinacionales y presentándolas como particulares afectados. Ambos, Estado y multinacionales, se reúnen con asiduidad, se premian y elogian públicamente por la política contra estos migrantes. Esta persecución se estructura a partir de la construcción de un perfil racial y se ejecuta con violencia institucional, dando cuenta del carácter estructural del racismo. Con lo cual son irregulares obligados a la subsistencia en la economía popular, pero su actividad laboral y su perfil racial los condenan a seguir siendo irregulares e, incluso, a ser expulsados del territorio. Como queda en evidencia, los derechos afectados no pueden analizarse por separado ya que intersectan dando forma a la construcción del colectivo senegalés como criminal. Esta situación se agravó y modificó notablemente desde diciembre del año 2015 cuando asumió la gestión el gobierno liberal-conservador de la Alianza Cambiemos. En este trabajo se evidenció este hecho a través de las presentaciones de organizaciones sociales y de derechos humanos ante el Estado y en los informes del Sistema Internacional de Derechos Humanos sobre este período. 


\section{BIBLIOGRAFÍA}

ACNUR. Refúgio no Brasil: Uma Análise Estatística: Janeiro de 2010 a Outubro de 2014. Brasília, 2014. Disponible en: https://reporterbrasil.org.br/ documentos/17nov2014__factsheet.pdf. Acceso en: 3 set. 2021.

ALBERDI, J. B. Peregrinación de Luz del Día: o viaje y aventuras de la Verdad en el nuevo mundo. Buenos Aires: Editorial La Cultura Argentina, 1916.

AMNISTIA INTERNACIONAL. Agenda para Argentina. 2017. Disponible en: https://amnistia.org.ar/wp-content/uploads/delightful-downloads/2017/01/ AGENDA-2017.pdf?utm_source=Prensa\&utm_campaign=98c64349d0-EMAIL_ CAMPAIGN_2017_01_18\&utm_medium $=$ email $\& u t m \_t e r m=0 \_a 60 e 315 \mathrm{cac}-$ 98c64349d0-85404873. Acceso en: 13 set. 2021.

ARGENTINA. Poder Ejecutivo de la Nación. Decreto n 70/2017. Modificación de la ley $\mathrm{N}^{\circ}$ 25.871. Migraciones, Buenos Aires, 2017. Disponible en: http://www. migraciones.gov.ar/pdf_varios/residencias/Decreto_70-2017.pdf. Acceso en: 3 set. 2021.

ARGENTINA. Dirección nacional de migraciones. Disposición n 2: Régimen especial de regularización de extranjeros de nacionalidad senegalesa. 2013. Disponible en: https://www.argentina.gob.ar/normativa/nacional/disposici\%C3\%B3n-2-2013-20 7171. Acceso en: 3 set. 2021.

ARGENTINA. Ley n ${ }^{\circ}$ 25.871, del 17 de diciembre de 2003. Ley De Migraciones De La República Argentina. InfoLEG, Buenos Aires, 2003. Disponible en: http:// servicios.infoleg.gob.ar/infolegInternet/anexos/90000-94999/92016/texact.htm. Acceso en: 3 set. 2021.

ARGENTINA. Constitución de la Nación Argentina: Parte primera. Infojus, Buenos Aires, 1994.

BARATTA, A. Criminología crítica y política criminal alternativa. Derecho Penal y Criminologíá, Bogotá, v. 2, p. 41, 1979.

BHABHA, H. Nuevas minorías, nuevos derechos: Notas sobre cosmopolitismos vernáculos. Buenos Aires: Siglo XXI, 2013.

BIGO, D. Sécurité et immigration: vers une gouvernementalité par l'inquiétude?. Cultures \& Conflits, Paris, n.31-32, 1998. Disponible en: http://journals.open edition.org/conflits/539. Acceso en: 3 set. 2021.

BUENOS AIRES. Ministerio Público Fiscal. Distinción por la lucha contra el comercio ilegal. 2019. Disponible en: https://www.facebook.com/mpfcaba/posts/ 2553533848053127/. Acceso en: 31 ago. 2021. 
BUENOS AIRES. Defensoría del Pueblo. Desalojo en Once: la defensoría presente en la emergencia. 2018a. Disponible en: https://defensoria.org.ar/noticias/desalojoen-once-la-defensoria-presente-en-la-emergencia/. Acceso en: 3 set. 2021.

BUENOS AIRES. Ministerio de Justicia y Seguridad. Reconocimiento por la labor realizada por la Ley de Marcas. 2018b. Disponible en: https://www.buenosaires. gob.ar/justiciayseguridad/noticias/reconocimiento-por-la-labor-realizada-por-la-leyde-marcas. Acceso en: 31 ago. 2021.

BUENOS AIRES. Ministerio Público Fiscal. Segundo informe de gestión: Luis Cevasco. 2018c. Disponible en: https://es.calameo.com/accounts/1579241. Acceso en: 31 ago. 2021.

BUENOS AIRES. Ministerio Público de la Defensa. Programa contra la Violencia Institucional: Informe anual 2017a. Buenos Aires, 2017a. Disponible en: https:// www.mpd.gov.ar/index.php/publicaciones-violencia-institucional/363-unidadde-registro-de-hechos-de-tortura-y-otras-formas-de-violencia-institucional/4154informe-anual-2017. Acceso en: 31 ago. 2021.

BUENOS AIRES. Ministerio Público Fiscal. Reconocimiento a la Fiscal Celsa Ramírez. 2017b. Disponible en: https:/www.nuestrasvoces.com.ar/investigaciones/ celsa-ramirez-la-fiscal-de-nike-y-larreta/. Acceso en: 31 ago. 2021.

CÁMARA ARGENTINA DE COMERCIO Y SERVICIOS [CAC]. Informe de Venta Ilegal Callejera y Piratería en la Ciudad de Buenos Aires. Buenos Aires: CAC, feb. 2019. Disponible en: http://www.cac.com.ar/data/documentos/15_ VI-CAC\%20-\%20Febrero\%202019.pdf. Acceso en 3 set. 2021.

CARRIL, C. Informe técnico sobre el Decreto de Necesidad y Urgencia 70/17: Defensoría del Pueblo de la Ciudad Autónoma de Buenos Aires: Subdirección Migrantes. Buenos Aires, 2017.

CENTRO DE ESTUdios leGAleS Y SOCIALES [CELS]. Preocupación ante la escalada de violencia ejercida por el estado contra vendedores ambulantes senegaleses en la Ciudad de Buenos Aires. Buenos Aires: CELS, 2018. Disponible en: https://www.cels.org.ar/web/2018/06/preocupacion-ante-la-escalada-de-violenciaejercida-por-el-estado-contra-vendedores-ambulantes-senegaleses-en-la-ciudad-debuenos-aires/. Acceso en: 3 set. 2021.

CENTRO DE ESTUDIOS LEGALES Y SOCIALES [CELS]. Hostigados, violencia y arbitrariedad policial en los barrios populares. Buenos Aires: CELS, 2016. Disponible en: http://cels.org.ar/hostigados.pdf. Acceso en: 3 set. 2021. 
CERIANI CERNADAS, P. Nueva ley: un paso hacia una concepción distinta de la migración. In: GIUSTANINI, R. (comp.). Migración: un derecho humano. Buenos Aires: Prometeo, 2004. p. 113-135.

CIRIO, N. P. La desaparición del candombe argentino: Los muertos que vos matáis gozan de buena salud. Música e Investigación, Buenos Aires, n.12-13, p.181-202, 2003.

COMITÉ PARA LA ELIMINACIÓN DE LA DISCRIMINACIÓN RACIAL [CERD]. Observaciones finales sobre los informes periódicos $21^{\circ}$ a $23^{\circ}$ de la Argentina. 2016. Disponible en: https://www.mpd.gov.ar/pdf/CERD_C_ARG_ CO_21-23_26014_S.pdf. Acceso en: 31 ago. 2021.

CORAGGIO, J. L. Política, económica, comunicación, economía popular. Ecuador Debate, Quito, n.17, p.57-94, 1989.

CRENSHAW, K. W. Postscript. In: LUTZ, H.; HERRERA VIVAR, M. T.; SUPIK, L. (coord.). Framing Intersectionality: Debates on a Multi-Faceted Concept in Gender Studies. Farnham: Ashgate, 2011. p.221-233.

CRENSHAW, K. W. Mapping the margins: intersectionality, identity politics and violence against women of color. Stanford Law Review, Stanford, v.43, n.6, p.12411299, 1991.

CRENSHAW, K. W. Demarginalizing the intersection of race and sex: A black feminist critique of antidiscrimination doctrine, feminist theory and antiracist politics. University of Chicago Legal Fórum, Chicago, v.1989, n.1, art. 8, 1989. Disponible en: http://chicagounbound.uchicago.edu/uclf/vol1989/iss1/8. Acceso en: 3 set. 2021.

DISCURSO completo de Mauricio Macri ante la Asamblea Legislativa. La Nación, Buenos Aires, 2016. Disponible en: https://www.lanacion.com.ar/politica/discursocompleto-de-mauricio-macri-ante-la-asamblea-legislativa-nid1875715. Acceso en: 3 set. 2021.

DURANTE el gobierno de Macri se duplicaron las expulsiones de extranjeros. Tiempo Argentino, Buenos Aires, 2019. Disponible en: https://www.tiempoar.com. $\mathrm{ar} /$ nota/durante-el-gobierno-de-macri-se-duplicaron-las-expulsiones-de-extranjeros. Acceso en: 31 ago. 2021.

ECUADOR. Ministerio del Interior. Acuerdo Ministerial no 88. Quito, 2015. Disponible en: https://www.trabajo.gob.ec/wp-content/uploads/downloads/2015/04/ A.-M.-No.-0088-Norma-que-regula-los-contratos-individuales-de-trabajo-a-plazofijo-y-de-enganche.pdf. Acceso en: 3 set. 2021. 
ECUADOR. Constitución de la República. Registro Oficial 449, de 20 de octubre de 2008. FielWeb, Quito, 2008. Disponible en: https://www.emov.gob.ec/sites/default/ files/transparencia_2018/a2.1.pdf. Acceso en: 3 set. 2021.

EL RECLAMO de los senegaleses llegó a la Legislatura: Entran a nuestras casas, nos golpean y nos roban todo. Nueva Ciudad, 2018. Disponible en: https://www. nueva-ciudad.com.ar/notas/201806/37750-el-reclamo-de-lossenegaleses-llego-a-lalegislatura-entran-a-nuestras-casas-nos-golpean-y-nos-robantodo.html. Acceso en: 31 ago. 2021.

ESPIRO, M. L.; ZUBRZYCKI, B. Tensiones y disputas entre migrantes africanos recientes y organismos de control estatal: El caso de los senegaleses en la ciudad de La Plata. Revista Question, Buenos Aires, v.1, n.39. p.109-121, 2013.

FUERTES enfrentamientos entre manteros senegaleses y la policía en Flores. Big Bang News, Buenos Aires, 12 abr. 2016. Disponible en: https://www.bigbangnews. $\mathrm{com} /$ actualidad/fuertes-enfrentamientos-entre-manteros-senegaleses-y-la-policia-enflores-2016-4-12-11-47-0. Acceso en: 3 set. 2021.

GARCÍA, M. I. ¿El racismo metamorfoseado? Acerca de los cambios recientes en torno a la negritud en la Argentina actual. Revista Kula, Buenos Aires, n.2, p.76-88, 2010 .

GOLDBERG, A.; SOW, P. Migrantes senegaleses en Argentina: contexto sociopolítico-laboral y vulneración de derechos. In: TEDESCO, J. C.; KLEIDERMACHER, G. (ed.). A imigração senegalesa no Brasil e na Argentina: múltiplos olhares. Porto Alegre: EST Ediçōes, 2017. p. 117-134.

INSTITUTO NACIONAL DE ESTADISTICAS Y CENSOS [INEC]. Anuario de entradas y salidas internacionales del Ecuador. Quito, 2019. Disponible en: https://www.ecuadorencifras.gob.ec/entradas-y-salidas-internacionales-2019/. Acceso en: 3 set. 2021.

JARAMILLO FONNEGRA, V. Cultura jurídica y mecanismos internacionales de protección de Derechos Humanos en la Argentina de Mauricio Macri (2016-2018). Revista Electrónica del Instituto de Investigaciones Ambrosio L. Gioja, Buenos Aires, n.22, p. 63-99, jun./nov. 2019.

KIMMEL, M. La producción teórica sobre la masculinidad: nuevos aportes. In: ASTELARRA, J. et al. Fin de siglo: genero y cambio civilizatorio. Santiago de Chile: Isis internacional, 1992. p. 129-138. (Ediciones de las mujeres, 17.).

KLEIDERMACHER, G. Representaciones sociales de migrantes senegaleses en Buenos Aires (1995-2014). Revista Mexicana de Sociología, Coyoacán, v. 79, n. 1, p. 65-92, 2017. 
KLEIDERMACHER, G. De la ilusión al desencanto: Senegaleses en Buenos Aires y la construcción de representaciones respecto a su proyecto migratorio. RUNA: Archivo para las Ciencias del Hombre, Buenos Aires, v.37, n.1, p. 89-104, 2016.

KLEIDERMACHER, G. Migración sur-sur: senegaleses en la Ciudad de Buenos Aires. Voces en el Fénix, Buenos Aires, v.21, p. 107-119, 2012.

LA LEGISLATURA porteña recibió denuncias de trabajadores senegaleses. La Izquierda Diario, 2018. Disponible en: https://www.youtube.com/watch?v=Z849 HenGX18. Acceso en: 3 set. 2021.

LACOMBA VÁZQUEZ, J. Inmigrantes senegaleses, islam y cofradías. Revista Internacional de Sociología, Córdoba, v. 59, n. 29, p. 163-187, 2018.

LYMAN, J. Estimaciones de la Población de Buenos Aires en 1744, 1778 y 1810. Desarrollo Económico, Buenos Aires, v.19, n.73, p.107-119, 1979.

MEGA operativo: Venta ilegal: 27 allanamientos simultáneos en Once y Flores. Clarín, Buenos Aires, 8 jun. 2018. Disponible en: https://www.clarin.com/ciudades/ venta-ilegal-27-allanamientos-simultaneos-onceflores_0_Sk9fc7OxX.html. Acceso en 3 set. 2021.

MÉNARD MARLEAU, A. Ecuador como nodo articulador de la migración senegalesa en América del Sur. Migración y Desarrollo, Zacatecas, v.15, n.29, p.31-50, 2017. Disponible en: http://www.redalyc.org/articulo.oa?id=66054713002. Acceso en: 3 set. 2021.

MENJIVAR OCHOA, M. Interseccionalidad de masculinidad, raza y clase: apuntes para un concepto de masculinidades neocoloniales. Tabula Rasa, Bogotá, n. 27, p. 353-373, 2017. Disponible en: http://dx.doi.org/10.25058/20112742.455. Acceso en: 3 set. 2021.

MIEMBROS de la CTEP e inmigrantes senegaleses fueron detenidos por la policía. Télam, 2017. Disponible en: https://www.telam.com.ar/notas/201711/223164detencion-operativo-ciudadanos-senegaleses-miembros-ctep-ciudad-de-buenos-aires. html. Acceso en: 31 ago. 2021.

MILlER, H. A. Race and Class Parallelism. The Annals of the American Academy of Political and Social Science, Thousand Oaks, CA, v.140, n.1, p.1-5, nov. 1928.

MONCLÚS MASÓ, M. La gestión penal de la inmigración: El recurso al sistema penal para el control de los flujos migratorios. 2005. 562f. Tesis (Doctorado en Sociologia Jurídico-Penal) - Facultat de Dret, Universitat de Barcelona, Barcelona, 2005. Disponible en: http://diposit.ub.edu/dspace/bitstream/2445/97667/1/ MONCLUS_MASÓ_TESIS.pdf. Acceso en: 31 ago. 2021. 
NACIONES UNIDAS. Grupo de Trabajo de Expertos sobre Afrodescendientes. Declaración a los medios del Grupo de Trabajo de Expertos de las Naciones Unidas sobre Afrodescendientes, al finalizar su visita oficial a la Argentina, realizada entre los días 11 y 18 de marzo de 2019. Buenos Aires, 2019. Disponible en: https://www.ohchr.org/SP/NewsEvents/Pages/DisplayNews.aspx?NewsID=24350\& LangI D=S Acceso en: 31 ago. 2021.

NACIONES UNIDAS. Consejo de Derechos Humanos. Informe del relator especial sobre las formas contemporáneas de racismo, discriminación racial, xenofobia y formas conexas de intolerancia. 2017. Disponible en: http://acnudh. org/wp-content/uploads/2017/08/racismo.pdf. Acceso en: 31 ago. 2021.

PACECCA, M. I.; CANELO, B.; BELCIC, S. Culpar a los negros ya los pobres: Los manteros senegaleses ante los allanamientos en el barrio de Once. In: PITA, M. V.; PACECCA, M. I. (ed.). Territorios de control policial: Gestión de ilegalismos en la Ciudad de Buenos Aires. Buenos Aires: Editorial de la Facultad de Filosofía y Letras, 2017. p. 199-219.

PENCHASZADEH, A. P.; GARCÍA, L. Política migratoria y seguridad en Argentina hoy: ¿el paradigma de derechos humanos en jaque? / Migration policy and security in Argentina today: human rights paradigm in jeopardy?. URVIO: Revista Latinoamericana De Estudios De Seguridad, Quito, n.23, p.91-109, 2018. Disponible en: https://doi.org/10.17141/urvio.23.2018.3554. Acceso en: 31 ago. 2021.

PÉREZ RABASA, J. Discriminación interseccional y criminalización de senegaleses en la Ciudad de Buenos Aires (2016-2019). 2019. 170f. Tesis (Maestría en Derechos Humanos) - Instituto de Justicia y Derechos Humanos Eduardo Luis Duhalde, Universidad Nacional de Lanús, Lanús, 2019.

PÉREZ RABASA, J. Migrantes en Argentina exigen derogación de decreto que los criminaliza. Pressenza: Internacional Press Agency, 2018. Disponible en: https:// www.pressenza.com/es/2018/04/migrantes-argentina-exigen-derogacion-decreto-loscriminaliza/. Acceso en: 31 ago. 2021.

REITER, P. Una aproximación etnográfica a las estrategias de inserción sociolaboral de migrantes senegaleses en Argentina. Buenos Aires, 2010. Ponencia presentada en las Primeras Jornadas de Estudios Afrolatinoamericanos.

RÍO ORTIZ, F. del. El lado negro de la Historia de Chile: El discurso historiográfico sobre los africanos y afrodescendientes durante el siglo XIX. 2009. 47f. Tesis (Doctorado en Ciencias Históricas) - Facultad de Filosofía y Humanidades, Universidad de Chile, Santiago, 2009. 
RUCHANSKY, E. El hotel de los africanos. Página 12, Buenos Aires, 12 mar. 2009. Disponible en: https://www.pagina12.com.ar/diario/sociedad/subnotas/3-38 888-2009-03-22.html. Acceso en: 31 ago. 2021.

SARUBBI, G. Una mirada antropológica sobre la migración senegalesa en Argentina, su inserción socio-laboral y la construcción de su identidad. 2014. Tesis de grado (Licenciatura en Antropología) - Universidad de Buenos Aires, Buenos Aires, 2014.

SEGATO, R. Racismo, discriminación y acciones afirmativas: herramientas conceptuales. In: ANSION, J.; TUBINO, F. (ed.). Educar en ciudadanía intercultural. Lima: Fondo Editorial de la Pontificia Universidad Católica del Perú, 2007. p.63-89. Disponible en: https://repositorio.pucp.edu.pe/index/bitstream/ handle/123456789/53738/Educar-en-ciudadania.pdf?sequence=1\#page=64. Acceso en: 31 ago. 2021.

SEGATO, R. La guerra contra las mujeres. Madrid: Traficante de sueños, 2016.

SOW, P. Prácticas comerciales transnacionales y espacios de acción de los senegaleses en España. In: ESCRIVÁ, A.; RIBAS, N. (coord.). Migración y Desarrollo: Estudios sobre remesas y otras prácticas transnacionales en España. Córdoba, España: Consejo Superior de Investigaciones Científicas, 2004. p. 235-254.

TEDESCO, J. C.; GRZYBOVSKI, D. Senegaleses no norte do Rio Grande do Sul: integração cultural, trabalho e dinâmica migratória internacional. Revista Espaço Pedagógico, Passo Fundo, v.18, n.2, 2011.

TENSIÓN con los manteros en Liniers: desalojaron 475 puestos ilegales. Infobae, 2018. Disponible en: https:/www.infobae.com/sociedad/2018/01/12/ operativopolicial-contra-los-manteros-en-liniers/. Acceso en: 3 set. 2021.

TREVISANI VESPA, M.; MAMBERTI, J. Sin vivienda para los más vulnerables en la Ciudad de Buenos Aires: Análisis de presupuesto y políticas públicas. Buenos Aires: Asociación Civil por la Igualdad y la Justicia (ACIJ), 2012. Disponible en: https://acij.org.ar/wpcontent/uploads/2012/12/Informe-vivienda-CORTO.pdf. Acceso en: 31 ago. 2021.

VIVEROS VIGOYA, M. La interseccionalidad: una aproximación situada a la dominación. Debate feminista, Coyoacán, n.52, p.1-17, 2016. Disponible en: doi. org/10.1016/j.df.2016.09.005. Acceso en: 31 ago. 2021.

ŽIŽEK, S. La nueva lucha de clases: Los refugiados y el terror. Barcelona: Anagrama, 2016. 
ZUBRZYCKI, B. La migración de los senegaleses Modou-Modou en Argentina. Contra Relatos desde el Sur, Buenos Aires, v.12, n.13, p.4-12, 2016.

ZUBRZYCKI, B. Nuevos destinos de la migración africana reciente: los senegaleses en Argentina. Travessia: Revista do Migrante, São Paulo, n.72, p.31-40, $2013 a$.

ZUBRZYCKI, B. Senegaleses en Argentina: redes, trayectorias y asociaciones. Colección UniCom, Buenos Aires, 2013b. Disponible en: https://ri.conicet.gov.ar/ bitstream/handle/11336/23667/CONICET_Digital_Nro.8b6ce35d6d25-46b1903f-1a0b339a63f0_B.pdf?sequence=5\&isAllowed=y. Acceso en: 31 ago. 2021.

ZUBRZYCKI, B. La migración senegalesa y la diáspora mouride en Argentina. Buenos Aires, 2009. Ponencia presentada en la VIII Reunión de Antropología del Mercosur.

Recebido em: 31 de agosto de 2020

Aprovado em: 28 de novembro de 2020 